\title{
Functional characterization of genetic variants in the porcine TLR3 gene
}

\author{
L. Wang' ${ }^{1,2}$, Y.C. Chen ${ }^{1}$, D.J. Zhang' ${ }^{2}$ H.T. Li ${ }^{1}$, D. Liu' ${ }^{2}$ and X.Q. Yang ${ }^{1}$ \\ ${ }^{1}$ College of Animal Science and Technology, Northeast Agricultural University, \\ Harbin, China \\ ${ }^{2}$ Institute of Animal Husbandry, Heilongjiang Academy of Agricultural Sciences, \\ Harbin, China \\ Corresponding author: X.Q. Yang \\ E-mail: xiuqin163@163.com
}

Genet. Mol. Res. 13 (1): 1348-1357 (2014)

Received January 14, 2013

Accepted July 16, 2013

Published February 28, 2014

DOI http://dx.doi.org/10.4238/2014.February.28.7

\begin{abstract}
Toll-like receptor 3 (TLR3) recognizes double-stranded RNA, which is a molecular signature of viruses, and plays a pivotal role in host defense against viral invasion. Polymorphisms in the human TLR3 gene have been shown to affect the receptor function and to be associated with a variety of diseases, suggesting correlations between TLR3 polymorphisms and the disease resistance/susceptibility in pigs. In this study, 5 known non-synonymous single nucleotide polymorphisms (SNPs) in the coding sequences of the porcine TLR3 gene - c.800C $>\mathrm{T}$ (p.T267M), c.933A $>$ G (p.I311M), c.1116A $>$ T (p.K372N), c.2129C $>$ G (p.T710S), and c.2160T $>$ G (p.I720M) - were analyzed for their effect on receptor function in transiently transfected PK-15 cells by using a luciferase reporter assay. In addition, the distribution of SNP c.933A $>\mathrm{G}$ was analyzed among pig populations. SNP c.933A $>\mathrm{G}$ significantly decreased the response to poly(I:C) $(\mathrm{P}<0.05)$, as represented by the weaker induction of firefly luciferase relative to that achieved by wild-type TLR3. SNP c.933A $>$ G results in the alteration of conserved amino acids in the highly conserved segment of the 12th leucine repeat region and is conserved among TLR3 orthologs from fishes to primates.
\end{abstract}


Moreover, together with the results of previous studies, the results of the present study revealed that SNP c. $933 \mathrm{~A}>\mathrm{G}$ is found solely in local Chinese pig breeds. These results suggested that SNP c.933A $>$ G plays a role in porcine disease resistance/susceptibility.

Key words: Porcine; TLR3; Polymorphism; Receptor function

\section{INTRODUCTION}

The immune system, which consists of the innate and acquired immune systems, fights against invading microbial pathogens and defends hosts from infection. Activation of the immune system can be elicited by the recognition of pathogen-associated molecular patterns (PAMPs), structures conserved among microbial species, by germline-encoded patternrecognition receptors (PRRs) (Janeway and Medzhitov, 2002). The Toll-like receptor (TLR) family, comprising at least 12 functional members in mammals, is one of the most important PRRs and is essential for host defense against infection. Upon binding to PAMPs, TLRs trigger a series of signaling events, resulting in the induction of various cytokines and chemokines, which induce subsequent inflammation and immune responses (Akira et al., 2006; O’Neill and Bowie, 2007).

TLR3 recognizes double-stranded RNA (dsRNA), a product of viral multiplication. A synthetic dsRNA analog, poly(I:C), can activate TLR3 (Alexopoulou et al., 2001). After activation by dsRNA, TLR3 initiates the MyD88-independent signaling pathway either through the Toll/interleukin-1 receptor (TIR) domain-containing adaptor, which induces the interferon (IFN)- $\beta$ adaptor protein, TRIF, or through the TIR domain-containing adaptor molecule, TICAM-1, which leads to the activation and nuclear translocation of the transcription factors NF- $\kappa B$ and IRF3, ultimately inducing the production of type I IFNs such as IFN- $\beta$ and various proinflammatory cytokines (Yamamoto et al., 2002; Oshiumi et al., 2003).

Single nucleotide polymorphisms (SNPs) in TLR3 may affect receptor functions, including pathogen recognition and signaling and have been associated with host resistance/ susceptibility to diseases. For example, a non-synonymous SNP (c.1234C > T; p.P412L) in the coding sequences of the TLR3 gene significantly decreased the capacity of the receptor for binding dsRNA, and these SNPs have been associated with geographic atrophy (Yang et al., 2008; Zhou et al., 2011), subacute sclerosing panencephalitis (Ishizaki et al., 2008), recurrent herpes labialis (Yang et al., 2012), hepatitis B virus infection (Al-Qahtani et al., 2012), etc. In addition, an SNP $(-705 \mathrm{~A}>\mathrm{G})$ at the promoter region may predispose individuals to hepatitis $\mathrm{C}$ virus infection (Medhi et al., 2011). Furthermore, SNP -764G $>\mathrm{T}$ at the promoter region of grass carp was significantly associated with resistance to the grass carp reovirus (Heng et al., 2011). Therefore, TLR3 is a strong candidate gene for a number of diseases, not only in humans, but also in domesticated animals.

With respect to pigs, some studies have been conducted on TLR3 polymorphisms (Morozumi and Uenishi, 2009; Yang et al., 2011), but to the best of our knowledge, the functional relevance of these SNPs remains unknown. Here, the physiological effect of five known missense SNPs in the porcine TLR3 gene, c.800C $>$ T (p.T267M), c.933A $>$ G (p.I311M), c. $1116 \mathrm{~A}>\mathrm{T}$ (p.K372N), c.2129C $>$ G (p.T710S), and c.2160T $>$ G (p.I720M), on signal transduction was characterized in transiently transfected PK-15 cells. Additionally, the distribution 
of one SNP, c.933A $>\mathrm{G}$, was analyzed in pig populations. The results of this study will be useful for molecular marker characterization for breeding pigs with improved disease resistance.

\section{MATERIAL AND METHODS}

\section{Primers}

Primer pairs were designed based on the sequence deposited in GenBank (No. DQ266435) by using the Primer Premier 5.0 software. For cloning the complete coding sequence (CDS), a primer pair C1F/R, with enzyme restriction sites EcoRI and XhoI at the 5 '-end, respectively, was designed. For site-directed mutagenesis, two primers with a mismatched base at the target position were designed for each SNP. Additionally, an extra primer, $\mathrm{MR}$, containing the endonuclease recognition site, EcoRV, which is inherent to the CDS of the porcine TLR3 gene, was designed for mutant type (MT) eukaryotic expression construction. Detailed information of primer pairs is provided in Table 1.

\begin{tabular}{llc}
\multicolumn{2}{c}{ Table 1. Primers used in this study. } \\
\hline Primers & Sequence (5'-3') & Position* \\
\hline C1F & GAGAATTCATGAGCAGGAGTTTGCCTT & $1-19$ \\
C1R & CGCTCGAGTTAATGTACTGAATTTCTGGA & $2698-2718$ \\
M800F & ACCAGCAACAtGACTTTCTTTGG & $790-812$ \\
M800R & CCAAAGAAAGTCaTGTTGCTGGTTTGTA & $784-813$ \\
M933F & ACAATATaGAGCGTTTGTCTTCT & $926-948$ \\
M933R & ACGCTCATATTGTTATACTCCA & $917-939$ \\
M1116F & GCATAAAtAGGAATACTTTCACAGG & $1109-1133$ \\
M1116R & GAAAGTATTCCTaTTTATGCCTGG & $1105-1128$ \\
M2129F & TTCATGATAAgTGCCAGTATGCTACTGATTTT & $2119-2150$ \\
M2129R & ATCAGTAGCATACTGGCAcTTATCATGAAAAAG & $2110-2148$ \\
M2160F & TATgATACTGCTCATCCATTTCG & $2156-2179$ \\
M2160R & ATGGATGAGCAGTATCATAAAGAT & $2152-2175$ \\
MR & ATAAAAAGATATCCGCCAGCC & $2182-2202$ \\
\hline
\end{tabular}

*Location of primers in the coding sequence of the porcine TLR3 gene (GenBank No. DQ266435). Enzyme recognition sites are underlined. Mismatched bases are presented with small letters.

\section{Cloning}

Total RNA was isolated from spleen tissues of Yorkshire pigs with the Trizol reagent according to manufacturer specifications (Invitrogen, Carlsbad, CA, USA). cDNA was synthesized with $1 \mu \mathrm{g}$ total RNA using Superscript II and Oligo(dT) primers (Invitrogen). Polymerase chain reaction (PCR) was performed with the $\mathrm{C} 1 \mathrm{~F} / \mathrm{R}$ primer pair in a $25-\mu \mathrm{L}$ final volume using Ex Taq DNA polymerase (TaKaRa, Dalian, China), cDNA was synthesized, and the product was inserted into a pMD-18T vector (TaKaRa) for confirmation by sequencing. The recombinant pMD-18T vector carrying the complete CDS, named pMD-18T-WT-TLR3, was used for site-directed mutagenesis and wild-type (WT) eukaryotic expression vector construction.

\section{Site-directed mutagenesis}

Site-specific mutation was introduced using a PCR-based method as reported previ- 
ously (Li et al., 2011). Briefly, the mutation was introduced into the end of PCR products through two independent PCRs (Reactions A and B; Table 2) using primers containing mismatched bases, high-fidelity Pfu DNA polymerase (TransGen, Beijing, China), and pMD-18TWT-TLR3 as a template. Next, the two obtained overlapping PCR fragments were spliced to introduce the mutation into the products (Reaction C; Table 2). The products of Reaction $\mathrm{C}$ were sub-cloned into the $\mathrm{pMD}-18 \mathrm{~T}$ vector for sequencing confirmation.

Table 2. Primer pairs used in PCR-based site-directed mutagenesis.
\begin{tabular}{llll}
\hline Loci & Reaction $\mathrm{A}$ & Reaction $\mathrm{B}$ & Reaction C \\
\hline c. $800 \mathrm{C}>\mathrm{T}$ & $\mathrm{C} 1 \mathrm{~F} / 800 \mathrm{R}$ & $800 \mathrm{~F} / \mathrm{C} 1 \mathrm{R}$ & $\mathrm{C} 1 \mathrm{~F} / \mathrm{C} 1 \mathrm{R}$ \\
c. $933 \mathrm{~A}>\mathrm{G}$ & $\mathrm{C} 1 \mathrm{~F} / \mathrm{M} 933 \mathrm{R}$ & $\mathrm{M} 933 \mathrm{~F} / \mathrm{MR}$ & $\mathrm{C} 1 \mathrm{MR}$ \\
c. $1116 \mathrm{~A}>\mathrm{T}$ & $\mathrm{C} 1 \mathrm{~F} / \mathrm{M} 1116 \mathrm{R}$ & $\mathrm{M} 1116 \mathrm{~F} / \mathrm{MR}$ & $\mathrm{C} / \mathrm{MR}$ \\
c. $2129 \mathrm{C}>\mathrm{G}$ & $\mathrm{C} 1 \mathrm{~F} / 2129 \mathrm{R}$ & $2129 \mathrm{~F} / \mathrm{C} 1 \mathrm{R}$ & $\mathrm{C} 1 \mathrm{~F} / \mathrm{C} 1 \mathrm{R}$ \\
c. $2160 \mathrm{~T}>\mathrm{G}$ & $\mathrm{C} 1 \mathrm{~F} / 2160 \mathrm{R}$ & $2160 \mathrm{~F} / \mathrm{C} 1 \mathrm{R}$ & $\mathrm{C} 1 \mathrm{~F} / \mathrm{C} 1 \mathrm{R}$ \\
\hline
\end{tabular}

\section{Eukaryotic expression vector construction}

For construction of the WT eukaryotic expression vector, pMD-18T-WT-TLR3 and pcDNA3.1+ (Invitrogen) were double-digested with EcoRI and XhoI (TaKaRa), respectively; the target fragments were purified using the DNA Gel Extraction Kit (Watson, Shanghai, China), and were ligated using T4 DNA ligase (TaKaRa). After transformation into Escherichia coli JM109, the recombinant plasmids were extracted from bacterial colonies and identified by double-digestion using EcoRI and XhoI. Sequencing was performed by the Beijing Genomics Institute (China) for confirmation. The positive recombinant plasmids were named pcDNA3.1WT-TLR3. For construction of the MT expression vector, the pcDNA3.1-WT-TLR3 and the pMD-18T vector carrying mutant TLR3 were double-digested with EcoRI and XhoI (for 800, 2129, and 2160 loci) or EcoRI and EcoRV (for 933 and 1116 loci), respectively, and the target fragments were purified, ligated, and sequenced as described above.

\section{Transfection and dual-luciferase assay}

PK-15 cells were cultured as described previously (Li et al., 2011). The cells were transiently co-transfected with one of the TLR3 expression vectors (WT or MT), the IRF3dependent firefly luciferase construct (pISRE-Luc) (Stratagene, La Jolla, CA, USA), and the Renilla luciferase reporter plasmid (pRL-TK) (Promega, Madison, WI, USA) using the Lipofectamine 2000 reagent (Invitrogen). At $24 \mathrm{~h}$ post-transfection, cells were stimulated with poly(I:C). Firefly and Renilla luciferase activities were measured using the Dual-Glo Luciferase Assay System (Promega). The firefly luciferase activity was normalized by dividing it by the Renilla luciferase activity. To determine whether the difference between the WT and MT allele was significant, the Student $t$-test was performed using the SAS 9.1 software (SAS, Cary, NC, USA).

\section{Genotyping}

The distribution of SNP c. $933 \mathrm{~A}>\mathrm{G}$ in 42 Landrace pigs and in 220 individuals 
from a three-way crossbreed [Piétrain x (German Landrace x German Large White)] was analyzed. Genomic DNA was isolated from whole blood using the phenol-chloroform method. Genotyping was performed using the created restriction site PCR-restricted fragment length polymorphism method. Specifically, a mutant base was introduced into the amplification fragment by the primer to create a recognition site of the endonuclease $X b a \mathrm{I}$ with one kind of base, A, at the polymorphic site. The primer pair was as follows: 5'-tatttctttctggagtataacaatct- $3^{\prime}$ (forward; the underlined base represents the mismatched base) and 5'-tgcctggaaagttgttatct-3' (reverse). The PCR and digestion reactions were performed normally, and the subsequent fragment separation was conducted on $4 \%$ agarose gel for $1 \mathrm{~h}$ at $100 \mathrm{~V}$.

\section{RESULTS}

\section{SNPs of porcine TLR3}

Morozumi and Uenishi (2009) and Yang et al. (2011) previously searched for SNPs in CDS of the porcine TLR3 gene among different groups and identified 7 non-synonymous SNPs that led to amino acid substitutions (Table 3). SNPs c. $4 \mathrm{~A}>\mathrm{G}$ and c.95G $>$ A were present with very low frequencies, and in only one study among the 96 individuals from 11 breeds analyzed (Morozumi and Uenishi, 2009). Furthermore, SNPs c.800C $>$ T, c.933A $>$ G, and c.1116A $>$ T were present in the leucine rich repeat (LRR) domain. SNPs c. $1116 \mathrm{~A}>\mathrm{T}$ and c. $2129 \mathrm{C}>\mathrm{G}$ were prevalent alleles, being represented in $>10 \%$ of populations sampled by Morozumi and Uenishi (2009) and Yang et al. (2011), respectively. Additionally, SNP c.2160T $>$ G was identified at a high frequency (22.3\%) in populations consisting of 87 individuals of Chinese indigenous breeds and Western commercial pig breeds, and 10 Chinese wild boars (Yang et al., 2011); however, its frequency was lower (4.8\%) in a sample panel investigated by Morozumi and Uenishi (2009). LRR domains are involved in PAMPs recognition (Chang, 2010). In addition, the prevalence of SNPs suggests that they have potential to be used as molecular markers in pig breeding. Therefore, SNPs c.800C $>$ T (p.T267M), c.933A $>$ G (p.I311M), c.1116A $>$ T (p.K372N), c.2129C $>$ G (p.T710S), and c.2160T $>$ G (p.I720M) were selected for functional analysis in cultured cells.

\begin{tabular}{|c|c|c|c|c|c|}
\hline SNPs & Amino acid & Domain & Domain position & MAF (\%) $)^{1}$ & $\operatorname{MAF}(\%)^{2}$ \\
\hline c. $4 \mathrm{~A}>\mathrm{G}$ & p.S2G & $\mathrm{SP}$ & $1-24$ & 1.2 & - \\
\hline c. $95 \mathrm{G}>\mathrm{A}$ & p.R32K & LRRNT & $25-32$ & 0.6 & - \\
\hline c. $800 \mathrm{C}>\mathrm{T}$ & p.T267M & LRR10 & $251-276$ & 1.8 & - \\
\hline c. $933 \mathrm{~A}>\mathrm{G}$ & p.I311M & LRR12 & 301-324 & 0 & 4.6 \\
\hline c. $1116 \mathrm{~A}>\mathrm{T}$ & p.K372N & LRR14 & 358-381 & 16.7 & 41.38 \\
\hline c. $2129 \mathrm{C}>\mathrm{G}$ & p.T710S & $\mathrm{TM}$ & 705-724 & 21.4 & 12.64 \\
\hline c. $2160 \mathrm{~T}>\mathrm{G}$ & p.I720M & $\mathrm{TM}$ & 705-724 & 4.8 & 22.3 \\
\hline
\end{tabular}
investigated by Yang et al. (2011). Domains were predicted according to the description of Matsushima et al. (2007).

\section{Effect of SNPs on TLR3 function}

The complete CDS of the TLR3 gene in Yorkshire pigs was successfully obtained us- 
ing the reverse transcriptase PCR method. Compared with the sequence deposited in GenBank (No. DQ266435), 3 synonymous SNPs, c.36C $>$ T, c.2643T $>$ C, and c.2649A $>$ G, were identified. No missense mutation was found. The nucleotides at positions $800,933,1116,2129$, and 2160 were $\mathrm{C}, \mathrm{A}, \mathrm{A}, \mathrm{C}$, and $\mathrm{T}$, respectively, which are the most common nucleotide sequences among pig populations (Morozumi and Uenishi, 2009; Yang et al., 2011). Therefore, the sequence obtained was used as the WT TLR3 allele.

To determine whether the five SNPs resulting in amino acid substitutions affected TLR3 function, a cell-based assay was used to analyze TLR3-dependent activation of a firefly luciferase reporter, pISRE-Luc. pISRE-Luc contains direct repeats of the IFN-stimulated response element (ISRE) in the promoter region, which drives the expression of the downstream luciferase gene through binding activated IRF3. Therefore, the assay measures TLR3 function by the amount of firefly luciferase activity produced through activation of the IRF3 transcription factor. pRL-TK, constitutively expressing the Renilla luciferase, was used as a transfection control.

As shown in Figure 1, TLR3 responded to poly(I:C) in a dose-dependent manner with the best stimulation observed at $5 \mu \mathrm{g} / \mathrm{mL}$ for $12 \mathrm{~h}$. Furthermore, transiently transfected cells can produce weak luminescence without stimulation. Thus, in subsequent experiments, results were measured as fold-change in induction of firefly luciferase activity in the presence of poly(I:C) relative to the unstimulated control.

Upon stimulation with $5 \mu \mathrm{g} / \mathrm{mL}$ poly(I:C) for $12 \mathrm{~h}$, transfectants with c. $800 \mathrm{C}>\mathrm{T}$, c. $1116 \mathrm{~A}>\mathrm{T}$, c. $2129 \mathrm{C}>\mathrm{G}$, and c. $2160 \mathrm{~T}>\mathrm{G}$ mutants showed firefly luciferase activity comparable to that of the WT allele with no significant difference $(\mathrm{P}>0.05)$. Whereas, the transfectant with the c.933A $>$ G mutant showed a significant decrease in activity $(\mathrm{P}<0.05)$, which indicates weaker ability of the mutant protein to induce activation of IRF3 (Figure 2).

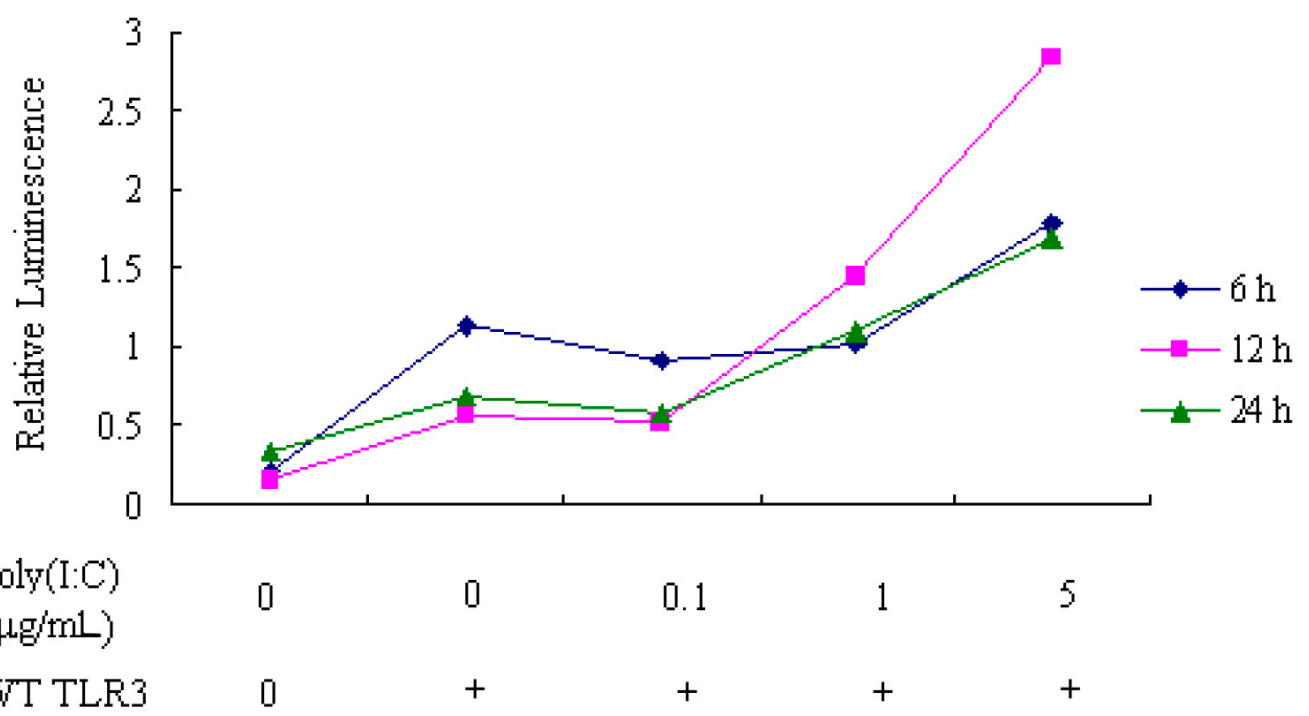

Figure 1. TLR3 responds to poly(I:C) in a dose-dependent manner in PK-15 cells. The values were measured as a ratio of firefly luciferase to that of Renilla luciferase. 


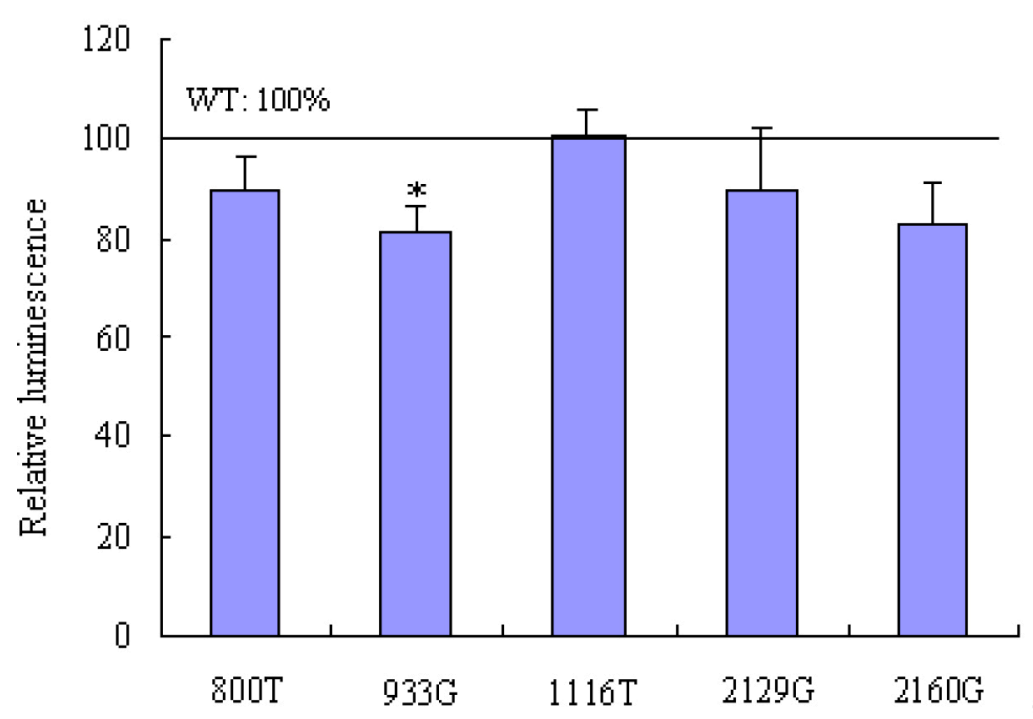

Figure 2. Effect of SNP in porcine TLR3 on the recognition of poly(I:C). The firefly luciferase activities of MT allele are displayed relative to that of the WT TLR3, which was normalized as $100 \%$. Data are reported as means \pm SE from three independent experiments, each with three replicates. Significant difference from the WT TLR3 was indicated $(* \mathrm{P}<0.05)$.

\section{Genotyping}

Morozumi and Uenishi (2009) and Yang et al. (2011) analyzed SNPs of the porcine TLR3 gene and their distribution among pig populations, and found that SNP c.933A $>\mathrm{G}$ was only present in local Chinese pig breeds, including Min and Beijing black pigs (Tables 3 and 4). To obtain further information of SNP c.933A $>$ G in Western commercial pig breeds, we genotyped Deutsche Landrace animals and individuals of a three-way crossbreed [Piétrain $\mathrm{x}$ (German Landrace $x$ German Large White)]. Our results showed that SNP c.933A $>$ G is rare in the two populations selected, because neither homozygote nor heterozygote carriers were found (Table 4).

Table 4. SNP c.933A $>$ G distribution among pig populations.
\begin{tabular}{lcccc}
\hline Allele & \multicolumn{3}{c}{ Frequency (\%) } \\
\cline { 2 - 5 } & $\mathrm{M}(30)^{*}$ & $\mathrm{~B}(12)^{*}$ & $\mathrm{~L}(42)$ & $\mathrm{C}(220)$ \\
\hline Allele A & 98.3 & 91.7 & 100 & 100 \\
Allele G & 1.7 & 8.3 & 0 & 0 \\
\hline
\end{tabular}

$\mathrm{M}=$ Min pig; $\mathrm{B}=$ Beijing black pig; $\mathrm{L}=$ Landrace; $\mathrm{C}=$ Crossbreed pig. *Data from Yang et al. (2011).

\section{DISCUSSION}

TLRs are type I transmembrane glycoproteins. Its ectodomain contains tandem LRRs, which have been suggested to be involved in ligand recognition (Chang, 2010). All LRRs 
contain a highly conserved segment (HCS) and a variable segment. The HCS is composed of an 11-residue stretch, LxxLxLxxNxL, in which "L" is L, I, V, or F, "N" is N, T, S, or C, and " $\mathrm{x}$ " is any amino acid (Kobe and Kajava, 2001). According to their cellular localization, TLRs can be divided into the cell-surface group (TLR1, 2, 4, 5, 6, and 10) and the cytoplasmic vesicle group (TLR3, 7, 8, and 9) (Chang, 2010). Compared with TLRs expressed on the cell surface, intracellular TLRs contain fewer non-synonymous SNPs in the protein-coding region (Shinkai et al., 2006; Morozumi and Uenishi, 2009). Only 4 and 7 non-synonymous SNPs were found in the human and porcine TLR3 gene, respectively (Ranjith-Kumar et al., 2007; Morozumi and Uenishi, 2009; Yang et al., 2011). Additionally, LRR domains of TLR3 and TLR7 show the lowest evolutionary rate from fishes to primates among all 15 TLRs identified thus far, except for TLR10 (Mikami et al., 2012). Together, this indicates that TLR3 has been subjected to severe selective pressures during evolution and any polymorphisms might therefore influence the receptor function. Genetic differences in the human TLR3 gene have been shown to influence the innate immune response through changing the receptor expression and/ or responsiveness to ligands, and have been associated with various diseases (Al-Qahtani et al., 2012; Clifford et al., 2012; Nuolivirta et al., 2012; Yang et al., 2012). These results suggest correlations between TLR3 polymorphisms and the disease resisitance/susceptibility in pigs.

In this study, we investigated 5 known non-synonymous SNPs, c. $800 \mathrm{C}>\mathrm{T}$, c. $933 \mathrm{~A}>\mathrm{G}$, c. $1116 \mathrm{~A}>\mathrm{T}, \mathrm{c} .2129 \mathrm{C}>\mathrm{G}$, and c. $2160 \mathrm{~T}>\mathrm{G}$, in the $\mathrm{CDS}$ of the porcine TLR3 gene for their functional relevance using a luciferase reporter assay in cultured PK-15 cells. One of the SNPs, c. $933 \mathrm{~A}>\mathrm{G}$, was found to significantly impair the responsiveness of the receptor to poly(I:C) $(\mathrm{P}<0.05)$, as represented by decreased production of IRF3. SNP c.933A $>$ G (p.I311M) results in the alteration of a conserved amino acid, the last "L", in the HCS of the 12th LRR (Matsushima et al., 2007). Moreover, I311 is conserved among TLR3 orthologs from fishes to primates (Figure 3). These observations suggest that I311 is crucial for the receptor function, which is consistent with our results.

\begin{tabular}{|c|c|c|}
\hline & LxxLxLxxHxL & \\
\hline Sus & LKYF F LE YNN IERLSSRSL YGLSN & 324 \\
\hline Homo & LE YF F LE YNN IQHLF SHSL HGLFN & 323 \\
\hline Mus & LRYLSLE YNN IORLSFRSF YGLSN & 324 \\
\hline Rattus & LKYLSLE YNN IQRL TP HSF RGLSN & 324 \\
\hline Bos & LEYLSLE YNN IE HLSSRSFYGLSN & 324 \\
\hline Equus & LE YF F LE YNN IE HL YUHSF YGLFN & 323 \\
\hline Felis & LEYF F LGMNN IE HLFSHSL YGLLS & 323 \\
\hline ovis & LE YLSLE YNN IE HLSSRSFYGLSS & 324 \\
\hline Pan & LE YF F LD YMN IQHLF SHSL HGLFN & 323 \\
\hline Macaca & LE YF F LE YNN IQHLLSHSL HGLFN & 323 \\
\hline Gallus & LE YLNLE DNN I INUSSHLFYGLSS & 323 \\
\hline Danio & LEF LSLE HNT IRHL TNDSFSGLGN & 324 \\
\hline
\end{tabular}

Figure 3. Alignment of the 11th LRR domain of TLR3 among species. p.311I in porcine was marked with asterisk. Arabic numerals indicate the position of the last base in the corresponding coding sequence. Homologous sequences were downloaded from GenBank with No. DQ266435 for Sus scrofa, NM003265 for Homo sapiens, NM198791 for Rattus norvegicus, NM001135928 for Ovis aries, NM126166 for Mus musculus, NM001079829 for Felis catus, NM001008664 for Bos taurus, NM001081798 for Equus caballus, EU204934 for Macaca mulatta, NM001130470 for Pan paniscus, NM001011691 for Gallus gallus, NM001013269 for Danio rerio. 
SNP c.933A $>\mathrm{G}$ was found solely in local Chinese pig breeds (Tables 3 and 4). Local Chinese and Western commercial pig breeds have different disease resistance owing to variations in their breeding environments. Local Chinese breeds, especially the Min pig, are famous for their high general resistance to disease. Therefore, this SNP might provide certain advantages to local Chinese pig breeds. However, the attenuating TLR signaling plays dual roles in host defense responses. For example, SNP c.1234C $>$ T in the human TLR3, which results in a reduced response to poly(I:C), is associated with susceptibility to recurrent herpes labialis on the one hand (Yang et al., 2012), whereas it confers natural resistance to HIV-1 infection and protects against geographic atrophy, on the other hand (Zhou et al., 2011; Sironi et al., 2012). Therefore, in order to determine to which disease SNP c.933A $>$ G originally provided the carrier resistance, further studies of specific virus infection are needed.

\section{ACKNOWLEDGMENTS}

Research supported by the National Natural Science Foundation of China (\#31072007) and the Doctoral Fund of the Ministry of Education of China (\#20112325120013).

\section{REFERENCES}

Akira S, Uematsu S and Takeuchi O (2006). Pathogen recognition and innate immunity. Cell 124: 783-801.

Al-Qahtani A, Al-Ahdal M, Abdo A, Sanai F, et al. (2012). Toll-like receptor 3 polymorphism and its association with hepatitis B virus infection in Saudi Arabian patients. J. Med. Virol. 84: 1353-1359.

Alexopoulou L, Holt AC, Medzhitov R and Flavell RA (2001). Recognition of double-stranded RNA and activation of NF-кB by Toll-like receptor 3. Nature 413: 732-738.

Chang ZL (2010). Important aspects of Toll-like receptors, ligands and their signaling pathways. Inflamm. Res. 59: 791-808.

Clifford HD, Yerkovich ST, Khoo SK, Zhang G, et al. (2012). TLR3 and RIG-I gene variants: associations with functional effects on receptor expression and responses to measles virus and vaccine in vaccinated infants. Hum. Immunol. 73: 677-685.

Heng J, Su J, Huang T, Dong J, et al. (2011). The polymorphism and haplotype of TLR3 gene in grass carp (Ctenopharyngodon idella) and their associations with susceptibility/resistance to grass carp reovirus. Fish Shellfish Immunol. 30: 45-50.

Ishizaki Y, Takemoto M, Kira R, Kusuhara K, et al. (2008). Association of Toll-like receptor 3 gene polymorphism with subacute sclerosing panencephalitis. J. Neurovirol. 14: 486-491.

Janeway CA Jr and Medzhitov R (2002). Innate immune recognition. Annu. Rev. Immunol. 20: 197-216.

Kobe B and Kajava AV (2001). The leucine-rich repeat as a protein recognition motif. Curr. Opin. Struct. Biol. 11: 725-732.

Li HT, Liu D and Yang XQ (2011). Identification and functional analysis of a novel single nucleotide polymorphism (SNP) in the porcine Toll-like receptor (TLR) 5 gene. Acta Agric. Scand. A Anim. Sci. 61: 161-167.

Matsushima N, Tanaka T, Enkhbayar P, Mikami T, et al. (2007). Comparative sequence analysis of leucine-rich repeats (LRRs) within vertebrate Toll-like receptors. BMC Genomics 8: 124.

Medhi S, Deka M, Deka P, Swargiary SS, et al. (2011). Promoter region polymorphism \& expression profile of Toll like receptor-3 (TLR-3) gene in chronic hepatitis C virus (HCV) patients from India. Indian J. Med. Res. 134: 200-207.

Mikami T, Miyashita H, Takatsuka S, Kuroki Y, et al. (2012). Molecular evolution of vertebrate Toll-like receptors: evolutionary rate difference between their leucine-rich repeats and their TIR domains. Gene 503: 235-243.

Morozumi T and Uenishi H (2009). Polymorphism distribution and structural conservation in RNA-sensing Toll-like receptors 3, 7, and 8 in pigs. Biochim. Biophys. Acta 1790: 267-274.

Nuolivirta K, He Q, Vuononvirta J, Koponen P, et al. (2012). Toll-like receptor 3 L412F polymorphisms in infants with bronchiolitis and postbronchiolitis wheezing. Pediatr. Infect. Dis. J. 31: 920-923.

O'Neill LA and Bowie AG (2007). The family of five: TIR-domain-containing adaptors in Toll-like receptor signalling. Nat. Rev. Immunol. 7: 353-364.

Oshiumi H, Matsumoto M, Funami K, Akazawa T, et al. (2003). TICAM-1, an adaptor molecule that participates in Tolllike receptor 3-mediated interferon-beta induction. Nat. Immunol. 4: 161-167. 
Ranjith-Kumar CT, Miller W, Sun J, Xiong J, et al. (2007). Effects of single nucleotide polymorphisms on Toll-like receptor 3 activity and expression in cultured cells. J. Biol. Chem. 282: 17696-17705.

Shinkai H, Tanaka M, Morozumi T, Eguchi-Ogawa T, et al. (2006). Biased distribution of single nucleotide polymorphisms (SNPs) in porcine Toll-like receptor 1 (TLR1), TLR2, TLR4, TLR5, and TLR6 genes. Immunogenetics 58: 324-330.

Sironi M, Biasin M, Cagliani R, Forni D, et al. (2012). A common polymorphism in TLR3 confers natural resistance to HIV-1 infection. J. Immunol. 188: 818-823.

Yamamoto M, Sato S, Mori K, Hoshino K, et al. (2002). Cutting edge: a novel Toll/IL-1 receptor domain-containing adapter that preferentially activates the IFN-beta promoter in the Toll-like receptor signaling. J. Immunol. 169: 6668-6672.

Yang CA, Raftery MJ, Hamann L, Guerreiro M, et al. (2012). Association of TLR3-hyporesponsiveness and functional TLR3 L412F polymorphism with recurrent herpes labialis. Hum. Immunol. 73: 844-851.

Yang XQ, Zhai CY, Li HT and Liu D (2011). Haplotype variation of Toll-like receptor among pig populations. Acta Agric. Scand. A Anim. Sci. 61: 7-11.

Yang Z, Stratton C, Francis PJ, Kleinman ME, et al. (2008). Toll-like receptor 3 and geographic atrophy in age-related macular degeneration. N. Engl. J. Med. 359: 1456-1463.

Zhou P, Fan L, Yu KD, Zhao MW, et al. (2011). Toll-like receptor 3 C1234T may protect against geographic atrophy through decreased dsRNA binding capacity. FASEB J. 25: 3489-3495. 